TRANSACTIONS OF THE

AMERICAN MATHEMATICAL SOCIETY

Volume 330, Number 1, March 1992

\title{
THE GAUSSIAN MAP FOR RATIONAL RULED SURFACES
}

\author{
JEANNE DUFLOT AND RICK MIRANDA
}

\begin{abstract}
In this paper the Gaussian map $\Phi: \bigwedge^{2} H^{0}(C, K) \rightarrow H^{0}(C, 3 K)$ of a smooth curve $C$ lying on a minimal rational ruled surface is computed. It is shown that the corank of $\Phi$ is determined for almost all such curves by the rational surface in which it lies. Hence, except for some special cases, a curve cannot lie on two nonisomorphic minimal rational ruled surfaces.
\end{abstract}

\section{INTRODUCTION}

In this article we make a computation concerning the Gaussian map for curves lying on a minimal rational ruled surface. The reader may recall that the Gaussian map $\Phi$ for a curve $C$ maps $\wedge^{2} H^{0}\left(C, K_{C}\right)$ to $H^{0}\left(C, 3 K_{C}\right)$, and is defined locally as follows. Let $\sigma$ and $\tau$ be two global 1-forms on $C$. If $p \in C$, choose a local coordinate $z$ on $C$ at $p$. Then $d z$ generates the space of holomorphic 1-forms locally at $p$ as a module over the ring of holomorphic functions at $p$, and so we may write $\sigma=f(z) d z$ and $\tau=g(z) d z$, where $f$ and $g$ are holomorphic functions at $p$. With this notation the Gaussian map $\Phi$ evaluated at $\sigma \wedge \tau$ is given locally by

$$
\Phi(\sigma \wedge \tau)=\left[f^{\prime} g-g^{\prime} f\right] \cdot(d z)^{3},
$$

which the reader may check is a well-defined element of $H^{0}\left(C, 3 K_{C}\right)$, independent of the choice of local coordinate $z$.

If $C$ is canonically embedded in $\mathbb{P}^{g-1}$, the Gaussian map is the map on global sections corresponding to the Gauss map. I.e., if $G: C \rightarrow \operatorname{Gr}(1, g-1)$ is the map sending a point of $C$ to its tangent line in $\mathbb{P}^{g-1}$, and $p: \operatorname{Gr}(1, g-1) \rightarrow$ $\mathbb{P}^{g(g-1) / 2}$ is the Plücker embedding of the Grassmann variety, then the Gaussian map $\Phi$ is the map determined by the composition $p \circ G$. The reader should consult [W1, W2, and C-H-M] for more details about the definition and basic properties of $\Phi$.

The most striking property which $\Phi$ has is its relationship to the deformation theory of the cone $X$ over the canonical image of $C$ in $\mathbb{P}^{g-1}$. In [W1], J. Wahl proves that the cokernel of $\Phi$ is exactly the $(-1)$-graded piece of the $T^{1}$ for $X$; his theorem is that if $\Phi$ is surjective, then the cone $X$ does not smooth (to a $K 3$ surface of degree $2 g-2$ in $\mathbb{P}^{g}$ ). It is not hard to see that $X$ being smoothable to a $K 3$ surface is equivalent to the curve $C$ occurring as a hyperplane section of a $K 3$ surface. Since "most" curves on a $K 3$ are very

Received by the editors January 22, 1990.

1980 Mathematics Subject Classification (1985 Revision). Primary 14H10, 14H45, 14J25, 14M20. 
ample (see [S-D]), we obtain the necessary condition that, loosely speaking, a curve with $\Phi$ surjective cannot lie on a $K 3$ surface. This is a quite interesting result, since the condition can be checked on the curve alone, without reference to any $K 3$ surface.

This article does not address the connection with curves on $K 3$ surface, but instead is a step towards addressing the question: how does the corank (=the dimension of the cokernel) of $\Phi$ stratify the moduli space for curves of genus $g$, and in particular what geometric meaning can be given to curves having certain given values for the corank of $\Phi$ ? Although we by no means get near to an answer to these questions, our computations are aimed in this direction.

In $\S 2$ of this paper we define a Gaussian map for line bundles on a surface, and show that if a curve $C$ lies on a surface $S$, then the Gaussian map for $C$ can be viewed as the restriction to $C$ of a certain Gaussian map on $S$. We then give a lemma (Lemma 2.6) allowing one to compute the corank of the Gaussian map for the curve $C$ in terms of data on the surface $S$.

We intend to apply this lemma in the case that the surface is one of the minimal rational ruled surfaces $\mathbb{F}_{n}$. In $\S 3$ we make the cohomology computations for the relevant vector bundles on $\mathbb{F}_{n}$ which are needed to apply the lemma of $\S 2$.

$\S 4$ is the heart of the paper, where we prove the surjectivity of the Gaussian map on $\mathbb{F}_{n}$ associated to "big enough" divisors, and precisely determine how "big" is necessary.

In $\S 5$ we draw the corollaries of our computations for curves lying on these $\mathbb{F}_{n}$ 's. Firstly we are able to exhibit curves of a fixed genus with various coranks for their Gaussian maps. It was proved in [C-H-M] that the general curve of genus $g=10$ or $g \geq 12$ has a surjective Gaussian map: the corank is 0 . In [W1] and in [B-M] it is proved that if a curve lies on a $K 3$ surface, then the Gaussian map cannot be surjective: the corank is at least one. The maximum corank for a curve of genus $g$ is $3 g-2$, which is achieved by any hyperelliptic curve (see [W2 or C-M2]). Various other computations have been made in [C-M1 and C-M2], for curves of low genus, trigonal and elliptic-hyperelliptic curves, for certain cyclic covers of $\mathbb{P}^{1}$, and for certain curves on Del Pezzo surfaces. In $[\mathrm{M}]$, the second author has exhibited stable curves for which the Gaussian map has corank one. However we view this article as being the first attempt to systematically achieve a range of coranks.

Our second application is the following:

(1.1) Theorem. Let $C$ be a nonhyperelliptic, nontrigonal, and non-4-gonal curve. Then $C$ can be embedded into at most one $\mathbb{F}_{n}$ with $n \geq 3$.

This seems surprising to the authors, and we do not know if any result along these lines has been proved before. From the point of view of projective geometry, the surfaces $\mathbb{F}_{n}$ are realized as the rational normal scrolls $S_{a, b}$ of dimension 2 in $\mathbb{P}^{a+b+1}$; here $n=|a-b|$. Therefore the above theorem states that if a curve lies on two scrolls $S_{a_{1}, b_{1}}$ and $S_{a_{2}, b_{2}}$, with both $\left|a_{1}-b_{1}\right|$ and $\left|a_{2}-b_{2}\right|$ at least 3 , then $\left|a_{1}-b_{1}\right|=\left|a_{2}-b_{2}\right|$.

Of course, for high enough genus, the general curve lies on no $\mathbb{F}_{n}$, so from that point of view Theorem (1.1) is simply a refinement of this relatively easy statement. Using that approach, we see that a curve lying on an $\mathbb{F}_{n}$ must be special, and the above theorem can be recast to say that if a curve is so special 
that it lies on two different ones (with the indices at least 3 ), then it has to have a map to $\mathbb{P}^{1}$ of degree 4 or less.

\section{A LEMMA CONCERNING THE GAUSSIAN MAP FOR A CURVE ON A SURFACE}

Let $S$ be a smooth surface and $C \subset S$ a smooth curve on $S$. If $L$ is a line bundle on $C$, the Gaussian map for $L$ on $C$ is the map

$$
\phi_{C, L}: \bigwedge^{2} H^{0}(C, L) \rightarrow H^{0}\left(C, L^{2} \otimes \Omega_{C}^{1}\right)
$$

given in terms of a local coordinate $z$ on $C$ and a trivialization of $L$ by sending $f(z) \wedge g(z)$ to $\left[f^{\prime}-g f^{\prime}\right] d z$. This definition can be extended to the surface $S$ and a line bundle $M$ on $S$, giving a map

$$
\phi_{S, M}: \bigwedge^{2} H^{0}(S, M) \rightarrow H^{0}\left(S, M^{2} \otimes \Omega_{S}^{1}\right)
$$

defined in almost the same way: if $\{z, w\}$ are local coordinates on $S$, then $f(z, w) \wedge g(z, w)$ is sent to $f \cdot d g-g \cdot d f$.

One obtains the "canonical" Gaussian map for a curve $C$ by taking the line bundle $L$ to be the canonical bundle $\Omega_{C}^{1}$ of $C$; this will be denoted simply by $\phi_{C}: \bigwedge^{2} H^{0}\left(C, \Omega_{C}^{1}\right) \rightarrow H^{0}\left(C,\left(\Omega_{C}^{1}\right)^{\otimes 3}\right)$.

If we take the line bundle $M$ on $S$ to be $\Omega_{S}^{2} \otimes \mathscr{O}_{S}(C)=\mathscr{O}_{S}\left(K_{S}+C\right)$, then by the adjunction formula, $\left.M\right|_{C}=\Omega_{C}^{1}$ and we have a commutative diagram

$$
\begin{array}{c|cc}
\bigwedge^{2} H^{0}\left(S, \mathscr{O}_{S}\left(K_{S}+C\right)\right) & \stackrel{\phi_{S, M}}{\longrightarrow} & H^{0}\left(S, \mathscr{O}_{S}\left(2 K_{S}+2 C\right) \otimes \Omega_{S}^{1}\right) \\
& H^{0}\left(C,\left.\Omega_{S}^{1}\left(2 K_{S}+2 C\right)\right|_{C}\right) \\
\bigwedge^{2} H^{0}\left(C, \Omega_{C}^{1}\right) & \stackrel{\phi_{C}}{\longrightarrow} & H^{0}\left(C,\left(\Omega_{C}^{1}\right)^{\otimes 3}\right),
\end{array}
$$

where the vertical map Res on the left is simply restiction to $C$, and the vertical maps $\alpha$ and $\beta$ on the right are defined as follows.

By tensoring the sequence

$$
0 \rightarrow \mathscr{O}_{S}(-C) \rightarrow \mathscr{O}_{S} \rightarrow \mathscr{O}_{C} \rightarrow 0
$$

with $\mathscr{O}_{S}\left(2 K_{S}+2 C\right) \otimes \Omega_{S}^{1}$, we obtain the sequence

$$
\left.0 \rightarrow \Omega_{S}^{1}\left(2 K_{S}+C\right) \rightarrow \Omega_{S}^{1}\left(2 K_{S}+2 C\right) \rightarrow \Omega_{S}^{1}\left(2 K_{S}+2 C\right)\right|_{C} \rightarrow 0,
$$

which gives the map $\alpha: H^{0}\left(S, \Omega_{S}^{1}\left(2 K_{S}+2 C\right)\right) \rightarrow H^{0}\left(C,\left.\Omega_{S}^{1}\left(2 K_{S}+2 C\right)\right|_{C}\right)$. The dual of the normal bundle sequence for $C$ in $S$ is

$$
\left.0 \rightarrow N_{C / S}^{-1} \rightarrow \Omega_{S}^{1}\right|_{C} \rightarrow \Omega_{C}^{1} \rightarrow 0,
$$

and after tensoring this with $\left.\mathscr{O}_{S}\left(2 K_{S}+2 C\right)\right|_{C}=\left(\Omega_{C}^{1}\right)^{\otimes 2}$, and recalling that $N_{C / S}=\left.\mathscr{O}_{S}(C)\right|_{C}$, we have the sequence

$$
\left.\left.0 \rightarrow \mathscr{O}_{S}\left(2 K_{S}+C\right)\right|_{C} \rightarrow \Omega_{S}^{1}\left(2 K_{S}+2 C\right)\right|_{C} \rightarrow\left(\Omega_{C}^{1}\right)^{\otimes 3} \rightarrow 0,
$$

which gives the map $\beta: H^{0}\left(C,\left.\Omega_{C}^{1}\left(2 K_{S}+2 C\right)\right|_{C}\right) \rightarrow H^{0}\left(C,\left(\Omega_{C}^{1}\right)^{\otimes 3}\right)$.

The diagram (2.1) is adapted from a similar diagram occurring in [B-M, §2]. The idea of calculating $\Phi$ for a curve $C$ by using a Gaussian map on a variety containing $C$ already occurs in [W1, $\S 6]$. 
Note that Res is actually $\bigwedge^{2}$ res, where res: $H^{0}\left(S, \mathscr{O}_{S}\left(K_{S}+C\right)\right) \rightarrow H^{0}\left(C, \Omega_{C}^{1}\right)$ is also restriction to $C$. This map res comes from the short exact sequence

$$
0 \rightarrow \mathscr{O}_{S}\left(K_{S}\right) \rightarrow \mathscr{O}_{S}\left(K_{S}+C\right) \rightarrow \Omega_{C}^{1} \rightarrow 0,
$$

and therefore if $S$ is a regular $(q=0)$ surface, we have $H^{1}\left(S, \mathscr{O}_{S}\left(K_{S}\right)\right)=0$ and both res and Res are surjective. This proves the following.

(2.4) Lemma. If $S$ is a regular surface, then the rank of the Gaussian map $\phi_{C}$ for $C$ is equal to the rank of the composition $\beta \circ \alpha \circ \phi_{S, M}$, where $\alpha, \beta$, and $M$ are as above.

Let us turn our attention to the maps $\alpha$ and $\beta$. Note that from the long exact sequence for the sequence (2.2), we have that if $H^{1}\left(S, \Omega_{S}^{1}\left(2 K_{S}+C\right)\right)=0$, then $\alpha$ is surjective; this will be true if $C$ is "ample enough" on $S$. If in addition $H^{2}\left(S, \Omega_{S}^{1}\left(2 K_{S}+C\right)\right)=0$ and $H^{1}\left(S, \Omega_{S}^{1}\left(2 K_{S}+2 C\right)\right)=0$, then we must also have $H^{1}\left(C,\left.\Omega_{S}^{1}(2 K+2 C)\right|_{C}\right)=0$. In this case then the cokernel of $\beta$ is isomorphic to $H^{1}\left(C,\left.\mathscr{O}_{S}\left(2 K_{S}+C\right)\right|_{C}\right)$, using the long exact sequence for $(2.3)$.

By considering the sequence

$$
\left.0 \rightarrow \mathscr{O}_{S}\left(2 K_{S}\right) \rightarrow \mathscr{O}_{S}\left(2 K_{S}+C\right) \rightarrow \mathscr{O}_{S}\left(2 K_{S}+C\right)\right|_{C} \rightarrow 0,
$$

we see that if $H^{1}\left(S, \mathscr{O}_{S}\left(2 K_{S}+C\right)\right)$ and $H^{2}\left(S, \mathscr{O}_{S}\left(2 K_{S}+C\right)\right)$ are also both zero, then $H^{1}\left(C,\left.\mathscr{O}_{S}\left(2 K_{S}+C\right)\right|_{C}\right) \cong H^{2}\left(\mathscr{O}_{S}\left(2 K_{S}\right)\right)$, which by Serre duality is isomorphic to $H^{0}\left(S, \mathscr{O}_{S}\left(-K_{S}\right)\right)$. This is of course independent of $C$, and allows us to deduce the rank of $\beta$, given the other assumptions. We have therefore proved the following.

(2.6) Lemma. With the notations above, assume that

(a) $S$ is a regular surface,

(b) $H^{1}$ and $H^{2}$ of $\Omega_{S}^{1}\left(2 K_{S}+C\right)$ are zero,

(c) $H^{1}\left(\Omega_{S}^{1}\left(2 K_{S}+2 C\right)\right)$ is zero,

(d) $H^{1}$ and $H^{2}$ of $\mathscr{O}_{S}\left(2 K_{S}+C\right)$ are zero, and

(e) the Gaussian map $\phi_{S, M}$ is surjective, where $M=\mathscr{O}_{S}\left(K_{S}+C\right)$.

Then the corank of the Gaussian map $\phi_{C}$ for $C$ is $\operatorname{dim}_{\mathbb{C}} H^{0}\left(S, \mathcal{O}_{S}(-K)\right)$.

This dimension $h^{0}\left(S, \mathscr{O}_{S}(-K)\right)$ is the antigeometric genus (or possibly the geometric antigenus) of $S$ (the geometric genus being $\left.h^{0}\left(S, \mathscr{O}_{S}(+K)\right)\right)$.

The rest of this paper is concerned with verifying the hypotheses of the above lemma when $C$ is a "sufficiently ample" curve on one of the minimal rational ruled surfaces $\mathbb{F}_{n}$. These surfaces are certainly regular; they are rational. Moreover, they are all minimal surfaces except $\mathbb{F}_{1}$, which is the blowup of $\mathbb{P}^{2}$ at one point.

\section{THE COHOMOLOGY OF CERTAIN BUNDLES ON $\mathbb{F}_{n}$}

In this section, we calculate $H^{0}\left(\mathbb{F}_{n}, \mathscr{O}(D)\right)$ and $H^{0}\left(\mathbb{F}_{n}, \Omega^{1}(D)\right)$ for any divisor $D$ on $\mathbb{F}_{n}$. We shall use the following local description of $\mathbb{F}_{n}$ in the computations.

The surface $\mathbb{F}_{n}$ is covered by the following four charts, each of which is isomorphic to affine 2-space:

$U_{1}$, with affine coordinates $(x, u)$;

$U_{2}$, with affine coordinates $(x, v)$; 
$U_{3}$, with affine coordinates $(y, w)$; and

$U_{4}$, with affine coordinates $(y, z)$.

The relationships between the variables are:

$$
u=1 / v, \quad z=1 / w, \quad x=1 / y, \quad \text { and } u=1 / z y^{n} .
$$

In these coordinates, the map $\pi: \mathbb{F}_{n} \rightarrow \mathbb{P}^{1}$ giving $\mathbb{F}_{n}$ as a $\mathbb{P}^{1}$-bundle over $\mathbb{P}^{1}$ is $\pi(x, u)=\pi(x, v)=x ; \pi(y, w)=\pi(y, z)=1 / y$ (where $1 / 0$ means the point at infinity). We denote by $F$ the fiber over infinity; then $F$ does not appear in $U_{1}$ or $U_{2}$ and is given locally by $y=0$ in $U_{3}$ and $U_{4}$. Let the section $s$ of $\pi$ be given by $s(x)=(x, 0)$ in $U_{2}$ (if $x \neq \infty$ ) and $s(\infty)=(0,0)$ in $U_{4}$; we set $s\left(\mathbb{P}^{1}\right)=B$. Thus, $B$ does not appear in $U_{1}$ or $U_{3}$, and is given locally by $v=0$ in $U_{2}$ and $z=0$ in $U_{4}$.

If $f$ is a meromorphic section of a vector bundle on $\mathbb{F}_{n}$, then $f$ is in fact regular if and only if $\left.f\right|_{U_{1}}$ and $\left.f\right|_{U_{4}}$ are regular, since these two charts cover all but two points in $\mathbb{F}_{n}$.

The last fact that we need before we do the computations in this section is that the Picard group of $\mathbb{F}_{n}$ is freely generated by the classes of the fiber $F$ and the section $\mathrm{B}$; every divisor $D$ on $\mathbb{F}_{n}$ is linearly equivalent to $r B+s F$ for some integers $r$ and $s$ (see Hartshorne [H] or Griffiths-Harris [G-H]). Hence our task is to compute $H^{i}\left(\mathbb{F}_{n}, r B+s F\right)$ and $H^{i}\left(\mathbb{F}_{n}, \Omega^{1}(r B+s F)\right)$ for $i=0,1$, and 2 , for all $n \geq 0$, and for all $r$ and $s$.

\section{(3.2) Lemma.}

(3.2.1) $H^{0}\left(\mathbb{F}_{n}, \mathscr{O}(r B+s F)\right)$ is zero if either $r$ or $s$ is negative.

(3.2.2) If $r$ and $s$ are nonnegative, then $H^{0}\left(\mathbb{F}_{n}, \mathscr{O}(r B+s F)\right)$ is the linear span of $\left\{x^{i} u^{j} \mid i \geq 0,0 \leq j \leq r, 0 \leq i+n j \leq s\right\}$. Note that this is a set of independent monomials.

(3.2.3) $H^{0}\left(\mathbb{F}_{n}, \Omega^{1}(r B+s F)\right)$ is zero if either $r$ or $s$ is negative.

(3.2.4) If $r$ and $s$ are nonnegative, then $H^{0}\left(\mathbb{F}_{n}, \Omega^{1}(r B+s F)\right)$ is the linear span of the union of the following three disjoint sets whose elements are linearly independent:

$$
\begin{aligned}
& \mathscr{X}=\left\{x^{k} u^{l} d x \mid 0 \leq k, 0 \leq k+n l \leq s-2,0 \leq l \leq r\right\}, \\
& \mathscr{U}=\left\{x^{i} u^{j} d u \mid 0 \leq i, 0 \leq i+n(j+1) \leq s-1,0 \leq j \leq r-2\right\},
\end{aligned}
$$

and

$$
\mathscr{M}= \begin{cases}\left\{x^{s-1-n l} u^{l-1}(n u d x-x d u) \mid 1 \leq l \leq r-1 \text { and } 1+n l \leq s\right\} \quad \text { if } n>0, \\ \left\{x^{s} u^{j} d u \mid 0 \leq j \leq r-2\right\} \text { if } n=0 .\end{cases}
$$

Note that $\mathscr{X}$ (the dx terms) is empty if $0 \leq s \leq 1, \mathscr{U}$ (the du terms) is empty if $0 \leq s \leq n$ or $0 \leq r \leq 1$, and $\mathscr{M}$ (the mixed terms) is empty if $0 \leq r \leq 1$ or if $n>0$ and $0 \leq s \leq n$.

Proof. Since neither $B$ nor $F$ meet the chart $U_{1}$, an element of

$$
H^{0}\left(\mathbb{F}_{n}, \mathscr{O}(r B+s F)\right)
$$

(when restricted to $U_{1}$ ) is a regular function $f$ on $U_{1}$ and therefore is a polynomial $f(x, u)$ in the variables $x$ and $u$, since $U_{1}$ is isomorphic to $\mathbb{A}^{2}$. Using (3.1) to transform $f(x, u)$ to the chart $U_{4}$, we obtain $f\left(1 / y, 1 / y^{n} z\right)$; for this to be in $H^{0}\left(\mathbb{F}_{n}, \mathscr{O}(r B+s F)\right)$, its poles must be bounded by $r B+s F$. 
Therefore $z^{r} y^{s} f\left(y^{-1}, z^{-1} y^{-n}\right)$ must be a polynomial function on $U_{4}$; indeed, this $H^{0}$ may be identified with the space

$$
\left\{f(x, u) \in \mathbb{C}[x, u] \mid z^{r} y^{s} f\left(1 / y, 1 / y^{n} z\right) \in \mathbb{C}[x, u]\right\} .
$$

If either $r$ or $s$ is negative, this forces $f$ to be zero, proving (3.2.1).

Assume now that $r$ and $s$ are nonnegative. We may also assume that $f$ is a monomial: if $f$ is a polynomial, then $f$ satisfies the above condition if and only if all of its nonzero terms do. Let then $f(x, u)=x^{i} u^{j}$ for $i \geq 0$ and $j \geq 0$. Then $z^{r} y^{s}\left(y^{-i}\right)\left(y^{-n} z^{-1}\right)^{j}$ is a polynomial if and only if $i+n j \leq s$ and $j \leq r$; this proves (3.2.2).

An element of $H^{0}\left(\mathbb{F}_{n}, \Omega^{1}(r B+s F)\right.$ ) (when restricted to $U_{1}$ ) is a differential form $\omega=f(x, u) d x+g(x, u) d u$ where $f$ and $g$ are regular, and therefore polynomial, functions; transforming $\omega$ to the $U_{4}$ chart, we obtain $\omega=A(y, z) d y+B(y, z) d z$, where

$$
A(y, z)=-y^{-2} f\left(1 / y, 1 / y^{n} z\right)-n y^{-(n+1)} z^{-1} g\left(1 / y, 1 / y^{n} z\right),
$$

and

$$
B(y, z)=-y^{-n} z^{-2} g\left(1 / y, 1 / y^{n} z\right) .
$$

The form $\omega$ is in $H^{0}\left(\mathbb{F}_{n}, \Omega^{1}(r B+s F)\right)$ iff $z^{r} y^{s}[A(y, z) d y+B(y, z) d z]$ is a regular 1-form; which is true if and only if $z^{r} y^{s} A(y, z)$ and $z^{r} y^{s} B(y, z)$ are polynomials. Since $f$ and $g$ are polynomials, if either $r$ or $s$ is negative, $\omega$ cannot be regular; this proves (3.2.3).

To prove (3.2.4), assume that $r$ and $s$ are nonnegative. We can now check that the 1 -forms in the sets $\mathscr{X}$ and $\mathscr{U}$ are in $H^{0}\left(\mathbb{F}_{n}, \Omega^{1}(r B+s F)\right)$ by using the criterion above.

In particular, for $\omega=x^{k} u^{l} d x$, we have $f=x^{k} u^{l}$ and $g=0$, so $A=$ $-y^{-k-n l-2} z^{-l}$ and $B=0$; thus $\omega$ is in the space if and only if $r-l$ and $s-k-n l-2$ are both nonnegative. This yields the set $\mathscr{X}$.

For $\omega=x^{i} u^{j} d u, f=0$ and $g=x^{i} u^{j}$, so that $A=-n y^{-n-i-1-n j} z^{-1-j}$ and $B=-y^{-n-i-n j} z^{-2-j}$; thus $\omega$ is in the space if and only if $r-1-j$ and $s-i-1-n(j+1)$ are nonnegative. This leads to the set $\mathscr{U}$.

In general, the function $G(y, z)=z^{r} y^{s} B(y, z)=-z^{r-2} y^{s-n} g\left(y^{-1}, z^{-1} y^{-n}\right)$ is a polynomial if and only if $g \in H^{0}\left(\mathbb{F}_{n},(r-2) B+(s-n) f\right)$, by the analysis above. Therefore $g$ is in the span of $\left\{x^{i} u^{j} \mid i \geq 0,0 \leq j \leq r-2,0 \leq i+n j \leq\right.$ $s-n\}$, by (3.2.2). Note that this set contains all of the monomial coefficients in the set $\mathscr{U}$.

Assuming that $G(y, z)$ is a polynomial, then

$$
z^{r} y^{s} A(y, z)=-z^{r} y^{s-2} f\left(y^{-1}, z^{-1} y^{-n}\right)+n z y^{-1} G(y, z)
$$

is a polynomial if and only if

$$
H(y, z)=z^{r} y^{s-1} f\left(y^{-1}, z^{-1} y^{-n}\right)-n z G(y, z)
$$

is a polynomial multiple of $y$. In particular this implies that $K(y, z)=$ $z^{r} y^{s-1} f\left(y^{-1}, z^{-1} y^{-n}\right)$ must be a polynomial. This forces $f$ to lie in $H^{0}\left(\mathbb{F}_{n}, r B+(s-1) F\right)$, and therefore $f$ is in the span of $\left\{x^{k} u^{l} \mid k \geq 0\right.$, $0 \leq l \leq r, 0 \leq k+n l \leq s-1\}$, by (3.2.2). Note that this set contains all of the monomial coefficients in the set $\mathscr{X}$.

It therefore suffices to understand when $\omega=f d x+g d u$ is in

$$
H^{0}\left(\mathbb{F}_{n}, \Omega^{1}(r B+s F)\right)
$$


for $f$ in the span of the monomials $\left\{x^{k} u^{l} \mid k \geq 0,0 \leq l \leq r, k+n l=s-1\right\}$ and $g$ in the span of the monomials $\left\{x^{i} y^{j} \mid i \geq 0,0 \leq j \leq r-2, i+n j=s-n\right\}$.

With this reduction we may then write

$$
f(x, u)=\sum_{\substack{0 \leq l \leq r \\ n l \leq s-1}} a_{l} x^{s-n l-1} u^{l} \text { and } g(x, u)=\sum_{\substack{0 \leq j \leq r-2 \\ n(j+1) \leq s}} b_{j} x^{s-n(j+1)} u^{j} .
$$

In this case

$$
z^{r} y^{s} A(y, z)=-\sum_{\substack{0 \leq l \leq r, n l \leq s-1}} a_{l} z^{r-l} y^{-1}-n \sum_{\substack{0 \leq j \leq r-2 \\ n(j+1) \leq s}} b_{j} z^{r-j-1} y^{-1},
$$

and

$$
z^{r} y^{s} B(y, z)=-\sum_{\substack{0 \leq j \leq r-2 \\ n(j+1) \leq s}} b_{j} z^{r-j-2} .
$$

Hence there are no further conditions imposed by requiring $z^{r} y^{s} B$ to be a polynomial. The expression $z^{r} y^{s} A$ is a polynomial if and only if it is zero, since it is clearly a polynomial in $z$ divided by $y$. This is zero if and only if $a_{m}+n b_{m-1}=0$ for all $m$. In particular, since the index on the $b$ 's only goes from 0 to $r-2$, we must have $a_{0}=a_{r}=0$, and a basis for the solutions to these conditions is given by the set $\mathscr{M}$. This completes the proof of the lemma.

By counting the monomials allowed in $H^{0}\left(\mathbb{F}_{n}, r B+s F\right)$ using (3.2.2), and by counting the elements in the sets $\mathscr{X}, \mathscr{U}$, and $\mathscr{M}$, one arrives at the following.

(3.3) Corollary.

(3.3.1) Assume that either $r \geq 0$ and $s \geq n r$,

or $r=-1$ and $s$ is arbitrary,

or $n=0, r \geq-1$, and $s \geq-1$;

then $\operatorname{dim}_{\mathbb{C}} H^{0}\left(\mathbb{F}_{n}, r B+s F\right)=(r+1)(s+1)-n r(r+1) / 2$.

(3.3.2) Assume that either $r \geq 1$ and $s \geq n r+2$,

or $n=0, r \geq 1$, and $s \geq 1$;

then $\operatorname{dim}_{\mathbb{C}} H^{0}\left(\mathbb{F}_{n}, \Omega^{1}(r B+s F)\right)=2 r s-n r^{2}-2$.

These computations of $h^{0}$ 's allow us to compute the $h^{2}$ 's in many cases, using Serre duality. The canonical class of $\mathbb{F}_{n}$ is $K_{\mathbb{F}_{n}}=-2 B-(n+2) F$, so that $h^{2}\left(\mathbb{F}_{n}, r B+s F\right)=h^{0}\left(\mathbb{F}_{n},(-r-2) B-(s+n+2) F\right)$. In addition, $h^{2}\left(\mathbb{F}_{n}, \Omega^{1}(r B+s F)\right)=h^{0}\left(\mathbb{F}_{n}, K_{\mathbb{F}_{n}} \otimes \mathbb{T}(-r B-s F)\right)$, where $\mathbb{T}$ is the tangent sheaf of $\mathbb{F}_{n}$, the dual to $\Omega^{1}$. However on a surface, $K \otimes \mathbb{T} \cong \Omega^{1}$, so that $h^{2}\left(\mathbb{F}_{n}, \Omega^{1}(r B+s F)\right)=h^{0}\left(\mathbb{F}_{n}, \Omega^{1}(-r B-s F)\right)$. In particular, using (3.2.1) and (3.2.3), we obtain the following.

(3.4) Corollary.

(3.4.1) If $r \geq-1$ or $s \geq-n-1$, then $H^{2}\left(\mathbb{F}_{n}, r B+s F\right)=0$.

(3.4.2) If $r \geq 1$ or $s \geq 1$, then $H^{2}\left(\mathbb{F}_{n}, \Omega^{1}(r B+s F)\right)=0$.

Now using the Riemann-Roch theorem for line bundles and rank 2 vector bundles on $\mathbb{F}_{n}$ we can determine the $h^{1}$ 's in the cases of interest.

We need to know the intersection numbers for divisors on $\mathbb{F}_{n}$; this is determined by the basic intersections $B \cdot B=-n, B \cdot F=1$, and $F \cdot F=0$. 
The Riemann-Roch theorem for line bundles tells us that

$$
\chi(D)=h^{0}\left(\mathbb{F}_{n}, D\right)-h^{1}\left(\mathbb{F}_{n}, D\right)+h^{2}\left(\mathbb{F}_{n}, D\right)=D(D-K) / 2+1+p_{a},
$$

and since the arithmetic genus of any $\mathbb{F}_{n}$ is zero we see that

$$
\begin{aligned}
\chi(r B+s F) & =\left[\left(-n r^{2}+2 r s\right)+(-2 n r+2 s+n r+2 r)+2\right] / 2 \\
& =(r+1)(s+1)-n r(r+1) / 2 .
\end{aligned}
$$

Therefore using Corollaries (3.3.1) and (3.4.1), we see the following.

(3.5) Lemma. If $r \geq 0$ and $s \geq n r$, or if $n=0, r \geq-1$, and $s \geq-1$, then $H^{1}\left(\mathbb{F}_{n}, r B+s F\right)=0$. 2.

Finally, consider the sheaf $\Omega^{1}(r B+s F)$, which is a locally free sheaf of rank

The Riemann-Roch theorem for a rank 2 bundle $\mathscr{E}$ over a surface $S$ tells us that

$$
\chi(\mathscr{E})=2 \chi\left(\mathscr{O}_{S}\right)-K_{S} c_{1} / 2+\left(c_{1}^{2}-2 c_{2}\right) / 2,
$$

where the $c_{i}$ 's are the Chern classes of $\mathscr{E}$.

In our case, $\chi(\mathscr{O})=1$, and if $\mathscr{E}=\Omega^{1}(D)$, then

$$
c_{1}=K+2 D \text { and } c_{2}=4+K \cdot D+D \cdot D .
$$

We then compute

$$
\begin{aligned}
\chi\left(\Omega^{1}(D)\right) & =2-\frac{1}{2}(K+2 D) K+\frac{1}{2}\left(K^{2}+4 D K+4 D^{2}-8-2 K D-2 D^{2}\right) \\
& =D^{2}-2 .
\end{aligned}
$$

If $D=r B+s F$, then $D^{2}-2=2 r s-n r^{2}-2$; this gives the following, using Corollaries (3.3.2) and (3.4.2).

(3.6) Lemma. If $r \geq 1$ and $s \geq n r+2$, or if $n=0, r \geq 1$, and $s \geq 1$; then $H^{1}\left(\mathbb{F}_{n}, \Omega^{1}(r B+s F)\right)=0$.

\section{THE SURJECTIVITY OF THE GAUSSIAN MAP FOR LINE BUNDLES ON $\mathbb{F}_{n}$}

In this section we compute the Gaussian map

$$
\Phi_{n, D}: \bigwedge^{2} H^{0}\left(\mathbb{F}_{n}, \mathscr{O}(D)\right) \rightarrow H^{0}\left(\mathbb{F}_{n}, \Omega^{1}(2 D)\right),
$$

for a divisor $D=r B+s F$ on $\mathbb{F}_{n}$; and determine conditions on $r, s$ and $n$ which give surjectivity of this map.

We immediately assume that $r$ and $s$ are nonnegative since our earlier calculations show that $\Phi_{n, D}$ is an isomorphism between trivial groups if either $r$ or $s$ is negative.

As we have calculated earlier,

$$
\text { domain } \Phi_{n, D}=\bigwedge^{2}\left\langle\left\{x^{i} u^{j} \mid i \geq 0,0 \leq j \leq r, 0 \leq i+n j \leq s\right\}\right\rangle .
$$

Also, range $\Phi_{n, D}=\langle\mathscr{X}\rangle+\langle\mathscr{U}\rangle+\langle\mathscr{M}\rangle$, where

$$
\begin{aligned}
\mathscr{X} & =\left\{x^{k} u^{l} d x \mid 0 \leq k, 0 \leq k+n l \leq 2 s-2,0 \leq l \leq 2 r\right\}, \\
\mathscr{U} & =\left\{x^{i} u^{j} d u \mid 0 \leq i, 0 \leq i+n(j+1) \leq 2 s-1,0 \leq j \leq 2 r-2\right\},
\end{aligned}
$$


and

$\mathscr{M}=\left\{\begin{array}{l}\left\{x^{2 s-1-n l} u^{l-1}(n u d x-x d u) \mid 1 \leq l \leq 2 r-1 \text { and } 1+n l \leq 2 s\right\} \quad \text { if } n>0, \\ \left\{x^{2 s} u^{l-1} d u \mid 1 \leq l \leq 2 r-1\right\} \text { if } n=0\end{array}\right.$ is

It is easy to see that the formula for $\Phi=\Phi_{n, D}$ on the pure monomial wedges

$$
\Phi\left(x^{\alpha} u^{\beta} \wedge x^{\gamma} u^{\delta}\right)=(\gamma-\alpha)\left(x^{\alpha+\gamma-1} u^{\beta+\delta} d x\right)+(\delta-\beta)\left(x^{\gamma+\alpha} u^{\beta+\delta-1} d u\right) .
$$

We first prove

(4.2) Lemma. If $s \geq n r$, then $\mathscr{M} \subseteq \operatorname{im} \Phi_{n, D}$.

Proof. Let $1 \leq l \leq 2 r-1$, and $s \geq n r$. Let

$$
c= \begin{cases}1 & \text { if } l \text { is odd } \\ 2 & \text { if } l \text { is even }\end{cases}
$$

Then

$$
\begin{gathered}
\Phi\left[(1 / c)\left(x^{s-n((l+c) / 2)} u^{(l+c) / 2} \wedge x^{s-n((l-c) / 2)} u^{(l-c) / 2}\right)\right] \\
=n x^{2 s-n l-1} u^{l} d x-x^{2 s-n l} u^{l-1} d u .
\end{gathered}
$$

Since $1+n l \leq 1+2 n r-n \leq 2 s+1-n$, if $n>0$ or if $n=0$, we have "hit" the typical element of $\mathscr{M}$; hence at this point we need only check that the above input to $\Phi$ is in fact in the domain. Certainly $(l \pm c) / 2 \geq 0$, and $s-n(l \pm c) / 2 \geq$ $s-n(l+c) / 2 \geq s-n r \geq 0$, so all exponents are positive. Moreover, it is clear that $(l \pm c) / 2 \leq r$. It remains to check that $[s-n(l \pm c) / 2]+n[(l \pm c) / 2] \leq s$; but it equals $s$ !

Next we consider $\mathscr{X}$.

(4.3) Lemma. If $s \geq n r+1$, then $\mathscr{X} \subseteq \operatorname{im} \Phi_{n, D}$.

Proof. Suppose $\mathscr{X} \neq \varnothing$; then $s \geq 1$. Fix $x^{k} u^{l} d x \in \mathscr{X}$; then $k \geq 0,0 \leq l \leq$ $2 r$, and $0 \leq k+n l \leq 2 s-2$.

Set $b=\min \{l, r\}$ and $c=\max \{0, k+n l+1-s\}$; note that $b$ and $c$ are both nonnegative.

Set $\alpha=\max \{c-n b, 0\}$ and $\beta=b$. Then

$$
\Phi\left[(1 / 2)\left(x^{\alpha} u^{\beta} \wedge x^{k-\alpha+1} u^{l-\beta}\right)-(1 / 2)\left(x^{\alpha+1} u^{\beta} \wedge x^{k-\alpha} u^{l-\beta}\right)\right]=x^{k} u^{l} d x .
$$

Again the problem is to verify that the element in the square brackets is indeed in the domain of $\Phi_{n, D}$.

Let us first check that all of the exponents above are nonnegative. It is clear that $\alpha$ and $\beta$ are at least 0 ; also $\beta \leq l$, so that $l-\beta \geq 0$. We need only check that $\alpha \leq k$, which is true if $\alpha=0$. If $\alpha>0$, then $\alpha=c-n b$, so that $c>0$; therefore $c=k+n l+1-s$. So $\alpha=c-n b=k+1-s$ if $b=l$; and $\alpha=c-n b=k+n(l-r)-(s-1)$ if $b=r$. If $b=l$, then since $s \geq 1$, we have $k-\alpha=s-1 \geq 0$; if $b=r$, then since $l \leq 2 r, k-\alpha=s+1-n(l-r) \geq s+1-n r$, which is nonnegative by assumption. Notice also that $l-\beta \leq r$.

To finish the proof, we must show that $\alpha+n \beta \leq s-1$, and that $k-\alpha+$ $n(l-\beta) \leq s-1$.

Case 1. $\alpha=0$ (i.e., $c \leq n b$ ). Then $\alpha+n \beta=n b \leq n r \leq s-1$. Also, $k+n l+1-s \leq c \leq n b=n \beta$, so that $k-\alpha+n(l-\beta) \leq s-1$. 
Case 2. $\alpha>0$ (i.e., $\alpha=c-n b$ and $n b \leq c$ ). In this case $\alpha+n \beta=$ $c-n b+n b=c$, and since $c>0, c=k+n l+1-s$. Since $k+n l \leq 2 s-2$, we have $c \leq s-1$, finishing the proof.

Finally, we consider $\mathscr{U}$.

(4.4) Lemma. If $s \geq n r+1$, then $\mathscr{U} \subseteq \operatorname{im} \Phi_{n, D}$.

Proof. Let $x^{i} u^{j} d u \in \mathscr{U}$, so that $i \geq 0,0 \leq j \leq 2 r-2$ and $0 \leq i+n(j+1) \leq$ $2 s-1$. Also, we may assume that $r \geq 1$ and $s \geq 1$, else $\mathscr{U}$ is empty.

Notice that if $i=0$, then

$$
u^{j} d u= \begin{cases}\Phi\left((1 /(j+1))\left(1 \wedge u^{j+1}\right)\right) & \text { if } j \leq r-1 \\ \Phi\left((1 /(j-2 r+1))\left(u^{r} \wedge u^{j-r+1}\right)\right) & \text { if } j \geq r-1\end{cases}
$$

Since $s \geq n r+1$, the indicated wedges are in the domain of $\Phi_{n, D}$. We may therefore assume that $i \geq 1$ from now on. Our analysis breaks into four cases:

(I) $0 \leq j \leq r-1$ and $i \leq s$;

(II) $0 \leq j \leq r-1$ and $i<s$;

(III) $r \leq j \leq 2 r-2$ and $n r \leq i+n(j+1)-s$;

(IV) $r \leq j \leq 2 r-2$ and $i+n(j+1)-s \leq n r$.

Case (I): $0 \leq j \leq r-1$ and $i \leq s$. One may calculate that

$$
\Phi\left((i-2)\left(u^{j+1} \wedge x^{i}\right)-(i)\left(x u^{j+1} \wedge x^{i-1}\right)\right)=(2 j+2) x^{i} u^{j} d u,
$$

and see that the indicated wedges are in the domain of $\Phi$.

Case (II): $0 \leq j \leq r-1$ and $i>s$. The formula

$$
\begin{aligned}
\Phi((2 s- & 2 n(j+1)-i-2)\left(x^{i+n(j+1)-s} \wedge x^{s-n(j+1)} u^{j+1}\right) \\
& -\left((2 s-2 n(j+1)-i)\left(x^{i+n(j+1)-s+1} \wedge x^{s-n(j+1)-1} u^{j+1}\right)\right) \\
= & (-2 j-2) x^{i} u^{j} d u
\end{aligned}
$$

works; the hypothesis that $i>s$ is only used to assure that the exponents of $x$ in the first terms of the two wedges are nonnegative.

Case (III): $r \leq j \leq 2 r-2$ and $i+n(j+1)-s \leq n r$. The formula

$$
\begin{gathered}
\Phi\left((i-2)\left(u^{r} \wedge x^{i} u^{j-r+1}\right)-(i)\left(x u^{r} \wedge x^{i-1} u^{j-r+1}\right)\right) \\
=(4 r-2 j-2) x^{i} u^{j} d u
\end{gathered}
$$

works.

Case (IV): $r \leq j \leq 2 r-2$ and $i+n(j+1)-s \geq n r$. Then

$$
\begin{aligned}
\Phi((2 s+ & 2 n r-2 n(j+1)-i-2)\left(x^{i+n(j+1)-s-n r} u^{r} \wedge x^{s+n r-n(j+1)} u^{j-r+1}\right) \\
& \left.-(2 s+2 n r-2 n(j+1)-i)\left(x^{i+n(j+1)-s-n r+1} u^{r} \wedge x^{s+n r-n(j+1)-1} u^{j-r+1}\right)\right) \\
= & (4 r-2 j-2) x^{i} u^{j} d u
\end{aligned}
$$

works.

This completes the proof of Lemma (4.4).

Lemmas (4.2)-(4.4) prove the following main result of this section. 
(4.5) Theorem. If $r \geq 0$ and $s \geq n r+1$, then $\Phi_{n, D}$ is surjective, where $D=$ $r B+s F$.

\section{Applications}

Our first task is to find the smooth curves $C$ on $\mathbb{F}_{n}$ which satisfy the conditions of Lemma (2.6). Since $\mathbb{F}_{n}$ is rational, it is regular, so (a) of Lemma (2.6) is satisfied.

Assume that $C$ is linearly equivalent to $p B+q F$ for some integers $p$ and $q$. Since $K_{\mathbf{F}_{n}}=-2 B-(n+2) F$, we see that

$$
\begin{aligned}
K+C & \equiv(p-2) B+(q-n-2) F, \\
2 K+C & \equiv(p-4) B+(q-2 n-4) F, \quad \text { and } \\
2 K+2 C & \equiv(2 p-4) B+(2 q-2 n-4) F .
\end{aligned}
$$

Therefore, by using (3.4.2) and Lemma (3.6), we see that

if $(p-4) \geq 1$ and $(q-2 n-4) \geq n(p-4)+2$, or if $n=0$ and both $p$ and $q$ are at least 5 , then $H^{1}$ and $H^{2}$ of $\Omega^{1}(2 K+C)$ both vanish, so that (b) of (2.6) is satisfied.

By using (3.6), we have

if $(2 p-4) \geq 1$ and $(2 q-2 n-4) \geq n(2 p-4)+2$, or if $n=0$ and both $p$ and $q$ are at least 3 , then $H^{1}$ of $\Omega^{1}(2 K+2 C)$ vanishes, so that (c) of (2.6) is satisfied.

By using (3.4.1) and (3.5), we see that

if $(p-4) \geq 0$ and $(q-2 n-4) \geq n(p-4)$, or if $n=0$ and both $p$ and $q$ are at least 3 , then $H^{1}$ and $H^{2}$ of $2 K+C$ vanishes, so that $(d)$ of $(2.6)$ is satisfied.

Finally, by Theorem (4.5), we have

if $p \geq 2$ and $(q-n-2) \geq n(p-2)+1$, then the Gaussian map

$\Phi$ for $K+C$ is surjective; hence (e) of (2.6) is satisfied.

Putting these all together, we obtain the following

(5.5) Theorem. Let $C$ be a smooth curve on $\mathbb{F}_{n}$, linearly equivalent to $p B+q F$. Assume that $p \geq 5$, and that

if $n=0$ then $q \geq 5$,

if $n=1$ then $q \geq p+4$, and

if $n=2$ then $q \geq 2 p+2$.

Then the dimension of the cokernel of the Gaussian map for $C$ is

$$
\begin{cases}9 & \text { if } n \leq 3 \\ n+6 & \text { if } n \geq 3\end{cases}
$$

Proof. Note that since $C$ is a smooth curve, we must have $q \geq n p$ (if $q<n p$ then every effective divisor linearly equivalent to $p B+q F$ meets $B$ negatively, and so must contain $B$ as a component). This inequality, along with the stated assumptions about $p$ and $q$, imply that the hypotheses of (5.1)-(5.4) are all true, so that all of the hypotheses of Lemma (2.6) hold. Therefore the corank of the Gaussian map for $C$ is $h^{0}\left(\mathbb{F}_{n}, \mathscr{O}(-K)\right)=h^{0}\left(\mathbb{F}_{n}, \mathscr{O}(2 B+(n+2) F)\right)$, which is 9 if $n \leq 2$ and is $n+6$ if $n \geq 3$. 
Our first application of the above theorem is to exhibit curves with a fixed genus, with various different coranks for their Gaussian maps. Note that by using the genus formula, one easily sees that the genus $g$ of a smooth curve on $\mathbb{F}_{n}$ linearly equivalent to $p B+q F$ is $g=(p-1)[q-1-n(p+2) / 2]$.

Therefore, if one fixes $g$, any solutions $(n, p, q)$ to the above equation with $p \geq 5$ and $q \geq n p$ will prove the existence of a smooth curve of genus $g$ with corank $(\Phi)$ given by the theorem: 9 if $n \leq 3$ and $n+6$ otherwise. (The condition $q \geq n p$ ensures that there are smooth curves in $|p B+q F|$ by Bertini's Theorem.)

For example, if $g$ is divisible by 4 , say $g=4 h$, then $p=5$ is possible; we then must have $2 q-2-7 n=2 h$, or $q=h+1-7 m$, with $n=2 m$, then $q \geq n p$ if and only if $h+1-7 m \geq 10 m$, or $m \leq(h+1) / 3$. This achieves even coranks $10,12,14, \ldots, 2[(h+1) / 3]+6$. In particular, if $g=100$, then $h=25$, so that even coranks from 10 through 22 are possible.

If $g$ is divisible by 5 , say $g=5 k$, then $p=6$ is possible; in this case $q=k+1+4 n$, so that we must have $k+1+4 n \geq 6 n$, or $n \leq(k+1) / 2$. In particular, if $g=100$, we have $k=20$; then the coranks $9,10, \ldots, 16$ are achieved.

The maximum corank for the Gaussian map of a curve of genus $g$ is achieved by a hyperelliptic curve (see [W2], or [C-M2]), and is $3 g-2$. We observe that in the above examples, we do not get very near to this bound; our results seem to obtain examples of "low" corank (although at least 9). It would be interesting to know, for a fixed genus, what coranks can occur: one conjecture is that anything between 0 and $3 g-2$ is possible (for $g \geq 12$ ).

Our second application is perhaps more interesting, and may have a wider audience. It follows by remarking that the corank of the Gaussian map for a curve as in Theorem (5.5) is independent of the curve, and only depends on the surface. Therefore, a curve (with certain restrictions) can lie on at most one $\mathbb{F}_{n}$. This seems a surprising result to the authors.

To be precise, recall that a curve is $k$-gonal if there exists a degree $k$ map from the curve to $\mathbb{P}^{1}$. A hyperelliptic curve is 2-gonal, and a trigonal curve is 3-gonal. We will say that a curve is non-k-gonal if such a map does not exist.

(5.6) Theorem. Let $C$ be a nonhyperelliptic, nontrigonal, and non-4-gonal curve. Then $C$ be embedded into at most one $\mathbb{F}_{n}$ with $n \geq 3$.

Proof. The hypotheses on $C$ imply that when $C$ is embedded into $\mathbb{F}_{n}$, it must be linearly equivalent to $p B+q F$, where $p \geq 5$. If $n$ is at least 3 , then $C$ and $n$ satisfy the hypotheses of Theorem (5.5); hence $n$ is determined (it must be 6 less than the corank of the Gaussian map for $C$ ).

\section{REFERENCES}

[B-M] A. Beauville and J.-Y. Mérindol, Sections hyperplanes des surfaces $K$ - 3 , Duke. Math. J. 55 (1987), 873-878.

[C-H-M] C. Ciliberto, J. Harris, and R. Miranda, On the surjectivity of the Wahl map, Duke Math. J. 57 (1988), 829-858.

[C-M1] C. Ciliberto and R. Miranda, On the Gaussian map for canonical curves of low genus, Duke Math. J. 61 (1990), 417-443.

[C-M2] _ _ Gaussian maps for certain families of canonical curves, Proc. Bergen 1989 Conf. in Algebraic Geometry (to appear). 
[G-H] P. A. Griffiths and J. Harris, Principles of algebraic geometry, Wiley, New York, 1978.

[H] R. Hartshorne, Algebraic geometry, Graduate Texts in Math., Vol. 52, Springer-Verlag, New York, 1977.

[M] R. Miranda, On the Wahl map for certain planar graph curves, (B. Harbourne and R. Speiser, eds.), Algebraic Geometry: Sundance 1988, Contemp. Math., vol. 116, Amer. Math. Soc., Providence, R.I., 1991, pp. 115-124.

[S-D] B. Saint-Donat, Projective models of K3 surfaces, Amer. J. Math. 96 (1974), 602-639.

[W1] J. Wahl, On the Jacobian algebra of a graded Gorenstein singularity, Duke Math. J. 55 (1987), 843-871.

[W2] _ Gaussian maps on algebraic curves, Preprint.

Department of Mathematics, Colorado State University, Fort Collins, Colorado 80523 